\title{
A Transient Enhancement Method for Two-Stage Helicopter Gearbox Fault Diagnosis Based on ALE
}

\author{
Xiange Tian", Tie Wang ${ }^{2}$, Zhi Chen ${ }^{2}$, Fengshou Gu${ }^{1}$, Andrew Ball ${ }^{1}$ \\ ${ }^{1}$ Centre for Efficiency and Performance Engineering, University of Huddersfield, Huddersfield, UK; ${ }^{2}$ Department of Vehicle Engi- \\ neering, Taiyuan University of Technology Taiyuan, Shanxi Province, China. \\ Email: F.Gu@hud.ac.uk,u1178848@hud.ac.uk
}

Received April, 2013.

\begin{abstract}
Periodical impulse component is one of typical fault characteristics in vibration signals from rotating machinery. However, this component is very small in the early stage of the fault and masked by various noises such as gear meshing components modulated by shaft frequency, which make it difficult to extract accurately for fault detection. The adaptive line enhancer (ALE) is an effective technique for separating sinusoidals from broad-band components of an input signal for detecting the presence of sinusoids in white noise. In this paper, ALE is explored to suppress the periodical gear meshing frequencies and enhance the fault feature impulses for more accurate fault diagnosis. The results obtained from simulated and experimental vibration signals of a two stage helical gearbox prove that the ALE method is very effective in reducing the periodical gear meshing noise and making the impulses in vibration very clear in the time-frequency analysis. The results show a clear difference between the baseline and 30\% tooth damage of a helical gear which has not been detected successfully in author's previous studies.
\end{abstract}

Keywords: Adaptive Filter; Adaptive Line Enhancement; Transient Enhancement; Fault Diagnosis

\section{Introduction}

Impulsive sound and vibration signals in machinery are often caused by component impacts which are commonly associated with component faults. It has long been recognized that the presence of a fault is often indicated by the presence, or increase in, impulsive signal elements. However, it tends to be difficult to make objective measurements of impulsive signals because of the high levels of background noise. The detection of these impulsive signals is hampered by the presence of the signals associated with the normal running of the machine, with the consequence that the detection of the weak impulsive signals, which are especially associated with incipient faults, is difficult [1]. It is the 'normal' signals which form the background noise environment against which the detection of fault induced impulsive signals must be conducted. To improve the precision of fault diagnosis, it is valuable to enhance the impulsive signals by suppressing this background noise prior to further processing.

De-noising and extraction of such faulty signals are very important for fault diagnostics, especially for early fault detection, in which the fault features are often very weak and embedded in noise. Therefore, it is necessary to enhance the data reliability and improve the accuracy of the signal analysis. After successful pre-processing the signal has an increased Signal to Noise Ratio (SNR), which makes it more amenable to one of a gamut of signal processing tools which can be used to characterize the signal, including Auto-Regressive (AR) modeling, kurtosis evaluation, cepstrum analysis, time-frequency analysis and higher order spectra analysis [2].

The ALE was introduced in as a method of detecting a periodic signal in an incoherent background or conversely of removing periodic interference from a broad-band signal of interest [3]. This technique can be used with any of the adaptive filters classified till now and uses a delay in the input signal to cancel out the unnecessary part in it and thus get the desired response. Naoto Sasaoka etc. applied ALE to reduce the sinusoidal noise in noise speech signal [4]. J. R. Mohammed etc. presented one noise reduction system based on two stages of operation with the first stage based on the ALE filters and the second stage on NLMS (Normalized Least Mean Square) filter. The first stage reduces the sinusoidal noise from the input signal and the second stage reduces the wideband noise [5]. S. K. Lee and P. R. White exploits two stage ALE filter structures in series to reduce the level of background noise. The resulting enhanced signals are analyzed in the time-frequency domain to obtain simultaneous spectral and temporal in- 
formation. The techniques developed are applied to the diagnosis of faults within an internal combustion engine and to data from an industrial gearbox [2].

In this paper, to reduce the stationary periodic gear meshing noise in gearbox vibration signals, ALE algorithm based on an adaptive LMS filter is examined for early fault detection. In such a way, the periodical noise is cancelled from the signal and impulses contain fault information are highlighted to produce more reliable detection and diagnosis results.

\section{Theoretical Background}

\subsection{Introduction of adaptive line enhancer}

Adaptive noise cancellation (ANC) is a procedure whereby a primary signal containing two uncorrelated components can be separated into those components by making use of a reference signal containing only one of them. The reference signal does not have to be identical to the corresponding part of the primary signal, just related to it by a linear transfer function. The ANC procedure adaptively finds that transfer function, and can thus subtract the modified reference signal from the primary signal, leaving the other component.

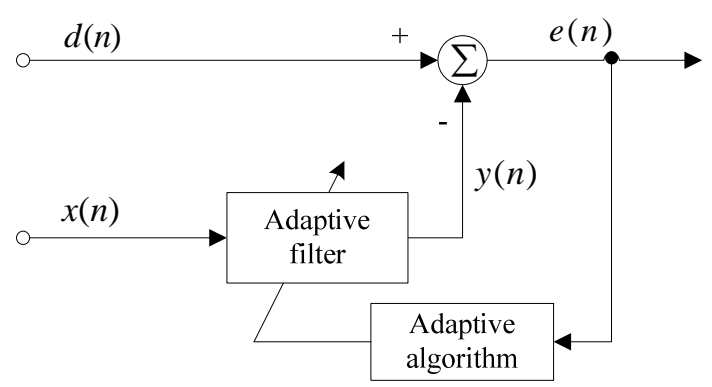

Figure 1. Block diagram of adaptive noise canceller.

A block diagram of an adaptive noise cancellation algorithm is shown in Figure 1. $d(n)$ is the primary input of the algorithm, $x(n)$ denotes the reference input to the adaptive filter and then filtered by the adaptive filter to produce an output $y(n)$ closely resembling $d(n) . y(n)$ is subtracted from $d(n)$ to produce the system output known as error signal $e(n)$. The adaptive filter coefficients are updated according to $e(n)$ and reference signal $x(n)$, which help minimize residual error noise.

ALE is in fact a degenerate form of the ANC in that its reference signal, instead of being derived separately, consists of a delayed version of the primary signal. According to Widrow [6] ALE is an adaptive self-tuning filter capable of separating the periodic and stochastic components in a signal different from other ANCs with multi-sensors. The ALE simply uses a single sensor and is therefore easier and more cost-effective to implement it in condition monitoring practice.

As shown in Figure 2, it is a simple variation of the ANC. It requires only a single input signal. In this case the reference signal is obtained by delaying the input signal by a certain number of samples $\Delta$. The adaptive filter then endeavors to predict the reference signal $x(n)$ from the delayed samples. The result is that any input components which are predictable over the delay appear at the filter output $y(n)$, whilst the error signal $e(n)$ contains those components which are unpredictable over the delay.

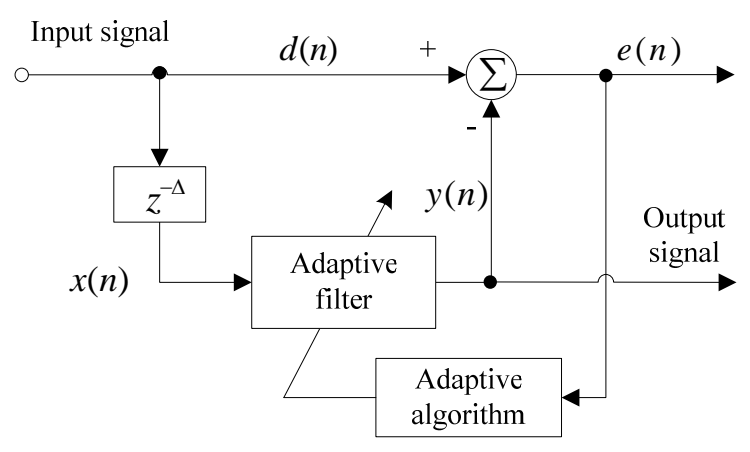

Figure 2. Block diagram of adaptive line enhancer.

The ALE becomes an interesting application in noise reduction because of its simplicity and ease of implementation. However, to obtain the best performance in its computational process, the optimal approach is to execute ALE on a better convergence rate of adaptive algorithm with a less complex adaptive filter structure. Adaptive algorithm could realize based on transversal Finite Impulse Response (FIR), recursive Infinite Impulse Response (IIR), lattice and sub-band filters [1].

The most widely used adaptive filtering technique is a version of the LMS algorithm, initially proposed by Widrow and Hoff. The LMS is based on the steepest descent method, a gradient search technique to determine filter coefficients that minimize the mean square prediction of a transversal filter. The NLMS converges faster than the conventional LMS because it employs a variable step size parameter aimed at minimizing the instantaneous output error [7, 8]. NLMS algorithm can be summarized as below.

The output of adaptive filter is

$$
y(n)=\mathbf{x}^{\mathbf{T}}(n) \mathbf{w}(n) .
$$

While $x(n)$ and $w(n)$ are given in Equation (2) and Equation (3)

$$
\begin{aligned}
& \mathbf{x}(n)=[x(n) \cdots x(n-L-1)]^{\mathbf{T}} \\
& \mathbf{w}(n)=\left[w_{0}(n) \cdots w_{L-1}(n)\right]^{\mathbf{T}}
\end{aligned}
$$

The adaptive coefficients update formula can be ex- 
pressed as

$$
\begin{gathered}
\mathbf{w}(n)=\mathbf{w}(n-1)-\frac{\mu}{\|\mathbf{x}(n)\|^{2}+\alpha} \mathbf{x}(n) e(n) \\
e(n)=d(n)-y(n)
\end{gathered}
$$

where the error signal $e(n)$ is given by Equation (5), $\mu$ is the step size parameter which controls the convergence speed and the stability of the filter and $\alpha$ is a small constant in order to prevent division by zero in case no input signal is present.

\subsection{Time Synchronous Average}

In experimental vibration, there are high levels of random noises which will affect the accuracy of fault diagnosis, especially random impulses which can confuse the periodicity of fault impulses. In this study, time synchronous average (TSA) technique is applied to suppress the random noises. TSA resamples the vibration data synchronously according to the angle of rotation. When taken over many machine cycles, this technique removes background noise and nonsynchronous events such as transients and meshing components from other gear transmission stages) in the vibration signal. This technique is extremely useful where multiple shafts those are operating at only slightly different speeds and in close proximity to one another are being monitored. A reference signal (usually from a tachometer) is always needed. However, it can be inefficient at reducing some tonal components of the background noise, i.e., those that are commensurate (or nearly commensurate) with the rotational frequency.

Assuming a signal $x(n)$ consists of a periodic signal $x_{T}(n)$ and a noisy component $v(n)$, the period of $x_{T}(n)$ is $T_{0}$ whose corresponding frequency is $f_{0}$, thus the signal can be expressed [9].

$$
x(n)=x_{T}(n)+v(n)
$$

The synchronous average of the signal $x(n)$ by using TSA can be expressed as

$$
y(n)=\frac{1}{M} \sum_{i=1}^{M-1} x\left(t+i T_{0}\right)
$$

where $M$ is the number of the average segments, $y(n)$ is the averaged signal.

\section{Signal Processing Methods}

\subsection{Denoising Scheme}

The principle of the ALE [10] used to reduce sinusoids will be described in this section based on the block diagram given in Figure 2.

The primary input and reference input signals of the system are given by

$$
\begin{gathered}
d(n)=s(n)+\eta(n) \\
x(n)=d(n-\Delta)=s(n-\Delta)+\eta(n-\Delta)
\end{gathered}
$$

where $s(n)$ is the fault impulse signal, $\eta(n)$ represents the sinusoidal noise and $\Delta$ is time delay factor. Since the short time duration of impulses, the autocorrelation of impulses fades as $\Delta$ increases. On the other hand, the delayed sinusoidal noise $\eta(n-\Delta)$ is correlated with $\eta(n)$. Thus, when the adaptive filter stable, $\eta(n)$ may be estimated by the output of adaptive filter $y(n)$ and the impulse components in error signal $e(n)$ are enhanced comparing with desired signal $d(n)$.

$$
\begin{aligned}
e(n) & =d(n)-y(n) \\
& =d(n)-\hat{\eta}(n) \\
& =s(n)+\eta(n)-\hat{\eta}(n)
\end{aligned}
$$

The cost function of NLMS algorithm is defined as

$$
\begin{aligned}
E\left[e^{2}(n)\right] & =E[d(n)-y(n)]^{2} \\
& =E[s(n)+\eta(n)-y(n)]^{2} \\
& =E[s(n)]^{2}+2 E[s(n)(\eta(n)-y(n))] \\
& +E[\eta(n)-y(n)]^{2}
\end{aligned}
$$

Since the fault signal is uncorrelated with noise, $E[s(n)(\eta(n)-y(n))]=0$. Then, Equation (11) becomes

$$
E\left[e^{2}(n)\right] \approx E[s(n)]^{2}+E[\eta(n)-y(n)]^{2}
$$

Minimize $E\left[e^{2}(n)\right]$ is equivalent to minimize $E[\eta(n)-y(n)]^{2}$. Therefore, the convergence of algorithm will cause $y(n)$ to be the minimum mean-square estimation of $\eta(n)$.

There are three important parameters in ALE, the step size $\mu$, the length of filter and the number of delay samples.

$\mu$ is the step size parameter controlling the convergence rate within its suitable range. The step size value affects the convergence behavior of an LMS filter; a too low value of $\mu$ leads to extremely long convergence time of the algorithm, whereas a too high value of $\mu$ causes the algorithm to diverge, thus degrading the error performance of the adaptive filter. Therefore, choosing a suitable value for the step size is necessary when implementing the LMS algorithm as an adaptive filter.

The periodic nature of the impulsive signal lays open the possibility that the first stage of the scheme will identify them with the narrowband components and in doing so attenuate them. To avoid such an eventuality care over the choice of the parameters $\Delta$ and $L$ must be exercised. From reference [5], the length of filter must satisfy the condition in Equation (14), where $T_{p}$ is the period of the 
signal.

$$
L<T_{p}-2 \Delta
$$

\subsection{Parameters Selection Method}

In this part, the selection method of ALE parameters applied in this paper is introduced.
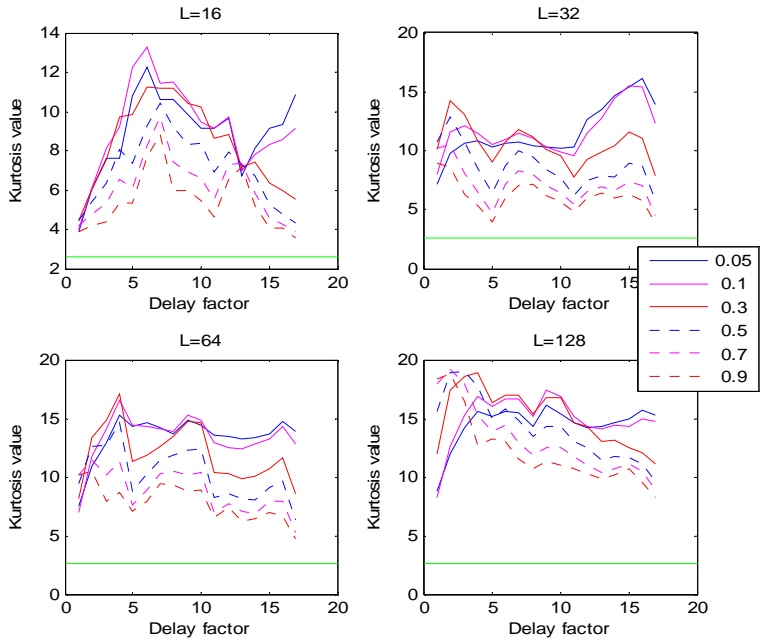

Figure 3. Kurtosis values under varies parameters.

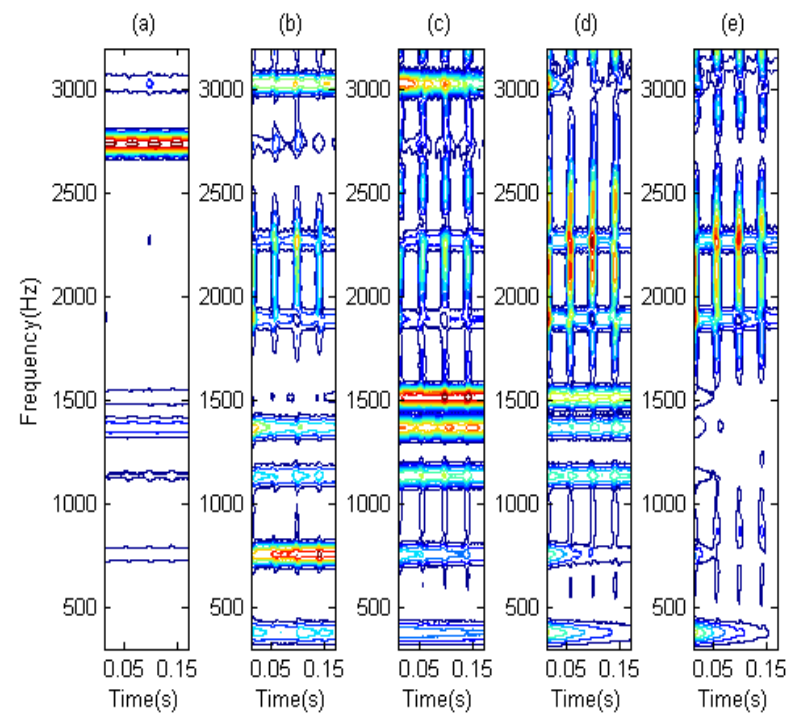

Figure 4. Time-frequency analysis results.

Kurtosis is used in engineering for detection of fault symptoms because it is sensitive to sharp variant structures, such as impulses. The bigger the impulse in signal, the larger the kurtosis is. The kurtosis value comparison of signals after ALE processing based on different parameter sets are shown in Figure 3. The green line indicates the kurtosis value of signal before ALE processing. Different line colors and style represent different $\mu$ values, $\mathrm{L}$ indicates the length of adaptive filter and delay factor is $\Delta$.

From Figure 3, it is obvious that kurtosis values are increased after ALE. To compare the impulse enhancement effects, the parameter sets which corresponding to maximum kurtosis under each $\mathrm{L}$ is selected for comparison. Then, the signals are analyzed in time-frequency domain.

Figure 4 illustrates the time-frequency analysis comparison between original signal and signals after ALE based on four different parameter sets. Figure 4(a) plots the original signal and Figures 4(b)-(e) give out the ALE results while the parameters are selected by maximum kurtosis criteria in Figure 3 under the four filter length 16, 32, 64 and 128, separately. It can be seen that the main periodic components at $1500 \mathrm{~Hz}$ and $2500 \mathrm{~Hz}$ are reduced greatly after ALE and the other components which are smaller can be observed clearly. It is obviously that the result in Figure 4(e), which corresponds to the maximum kurtosis value parameter set in Figure 3, can reveal the nonstationary components better than other three parameter sets. So the kurtosis maximization criterion is applied to choose ALE parameters in the following machine fault diagnosis.

\section{Signal Processing Results}

The practical gearbox experimental vibration signals collected when the gearbox operated under five different loads and four cases (Healthy, 30\% tooth break, 60\% tooth break and $100 \%$ tooth break).

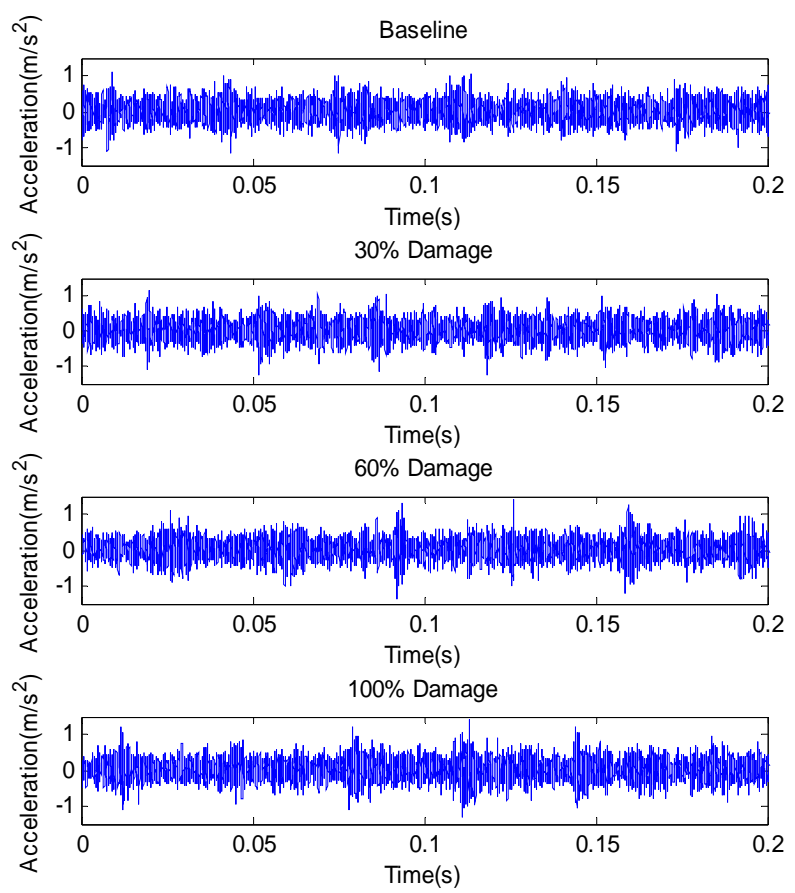

Figure 5. Raw vibration signal in time domain. 


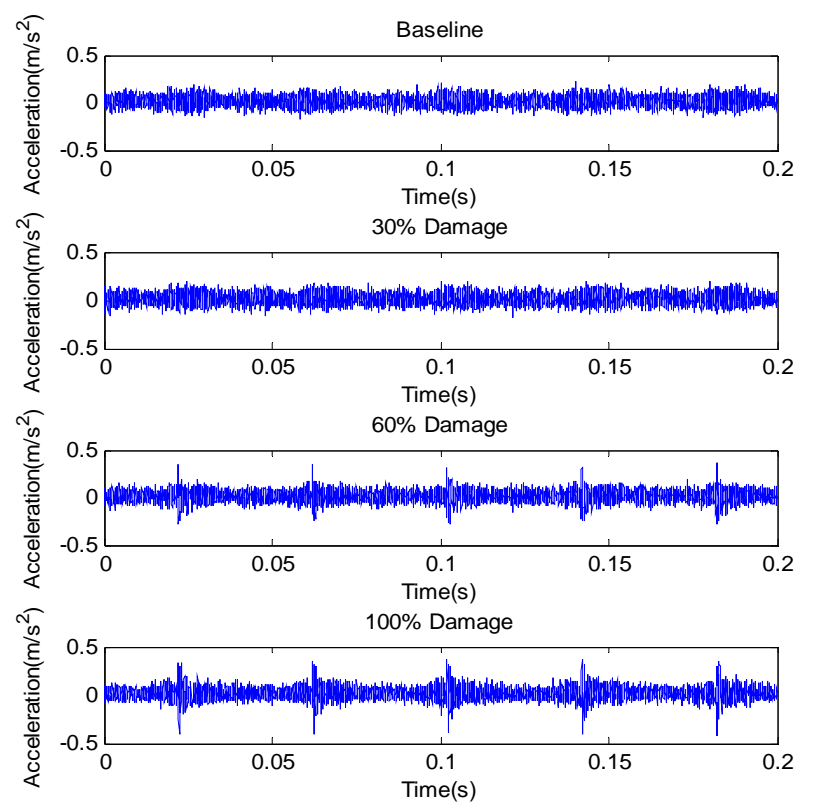

Figure 6. TSA vibration signal in time domain.

Figure 5 gives out the raw vibration data for four cases under load 3, where the baseline is the healthy case. Figure 6 shows the TSA results of the vibration signals given out in Fig. 3 for five revolutions of the input shaft. It can be seen that the impulse components of the vibration signals are highlighted for all the test conditions. TSA signals show much clearer indication of the $60 \%$ and $100 \%$ tooth damage compared with the baseline and $30 \%$ tooth damage. However the signals between the baseline and $30 \%$ tooth damage cannot be observed with noticeable differences.

To evaluate the denoise effect of ALE algorithm, Figure 7 and Figure 8 give out the time-frequency analysis results comparison, take the signal under the 4th load as example, signals under other loads have similar performance.

As shown in Figure 8, there are several periodical modulation frequencies generated by rotation of machine and can be seen as background noise at about $1500 \mathrm{~Hz}$, $3000 \mathrm{~Hz}$ and $4500 \mathrm{~Hz}$. The purpose of ALE is to remove these frequencies. The TSA signals also contain impulse components which are short duration and periodical contents for certain frequencies which are spread over the time direction. These components carry the fault information of gearbox tooth break damage. Figure 8 shows the results after the ALE processing. It can be seen that the modulation frequencies are reduced significantly. The results show that ALE is effective in reducing sinusoids and extract impulses from wide band periodical noises.

For a detailed comparison, two common feature parameters: root mean square (RMS) and kurtosis are calculated from the TSA vibration signals ALE errors sig- nals. As shown in Figure 9(a), RMS is not able to separate the four cases over different loads. Comparing with the baseline and 30\% tooth damage, in Figure 9(b), the kurtosis values of $60 \%$ tooth damage and $100 \%$ tooth damage is clearly separated from the other two cases except the first load. However, the difference between the baseline and $30 \%$ tooth damage is not very obvious for fault separation. Figure 9(c) and Figure 9(d) illustrate the RMS and kurtosis value comparison from the error signal which is the signal after ALE denoising processing. From the RMS value comparison of error signal, there are differences between all loads except the first and all four fault cases which gives out clear difference compared with TSA signal. The kurtosis value of error signal is much higher than that of TSA signal, when means the proportion of impulses is enhanced. But the difference between kurtosis values of different fault cases is not so clearly as the RMS value of error signal.

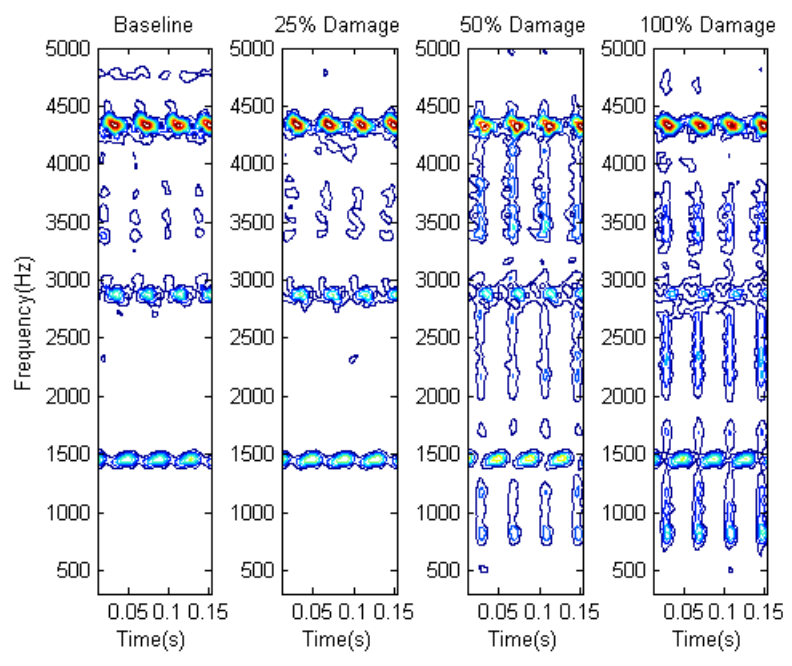

Figure 7. TSA vibration signals in time-frequency domain.

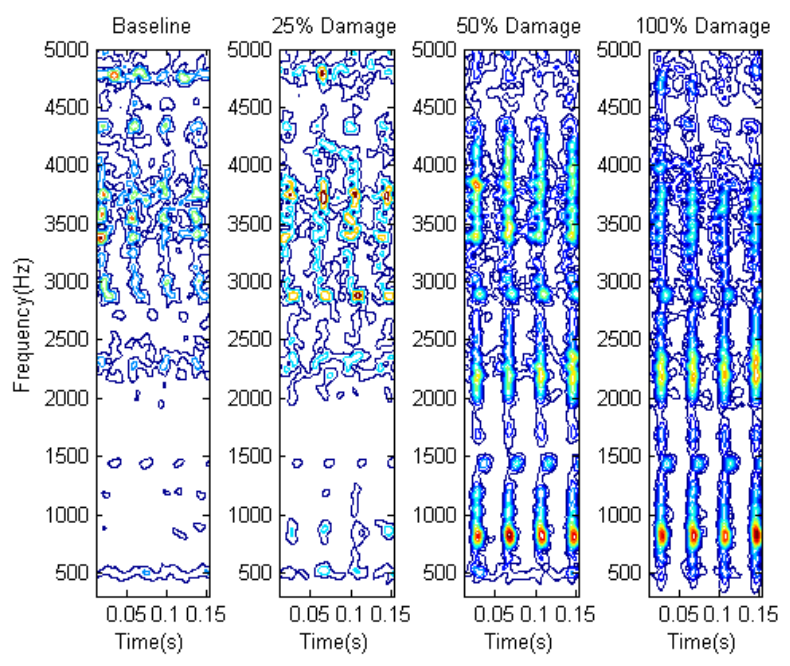

Figure 8. Signals after ALE in time-frequency domain. 

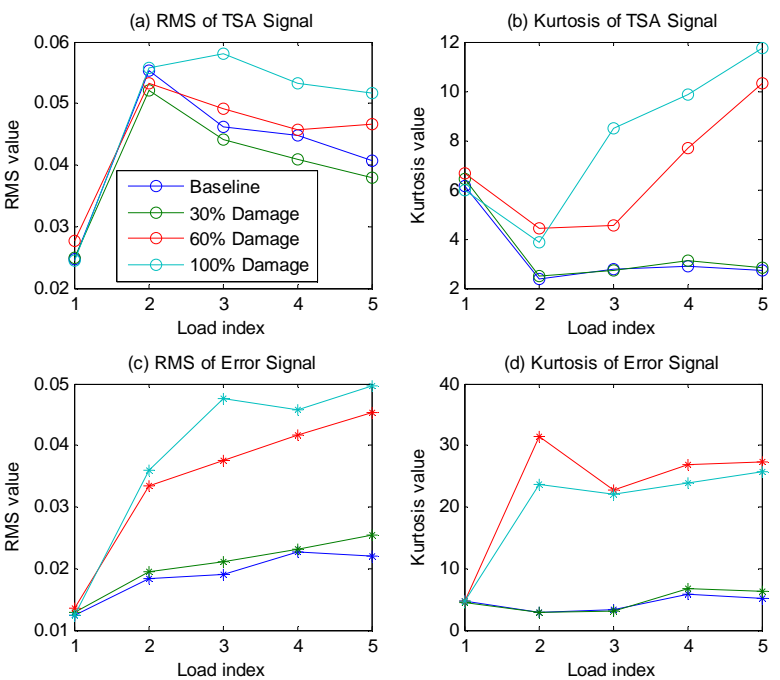

Figure 9. RMS and kurtosis value comparison of TSA and error signal $(\mathrm{L}=\mathbf{2 0}, \mathrm{mu}=\mathbf{0 . 0 7 5}, \Delta=\mathbf{1 2})$.
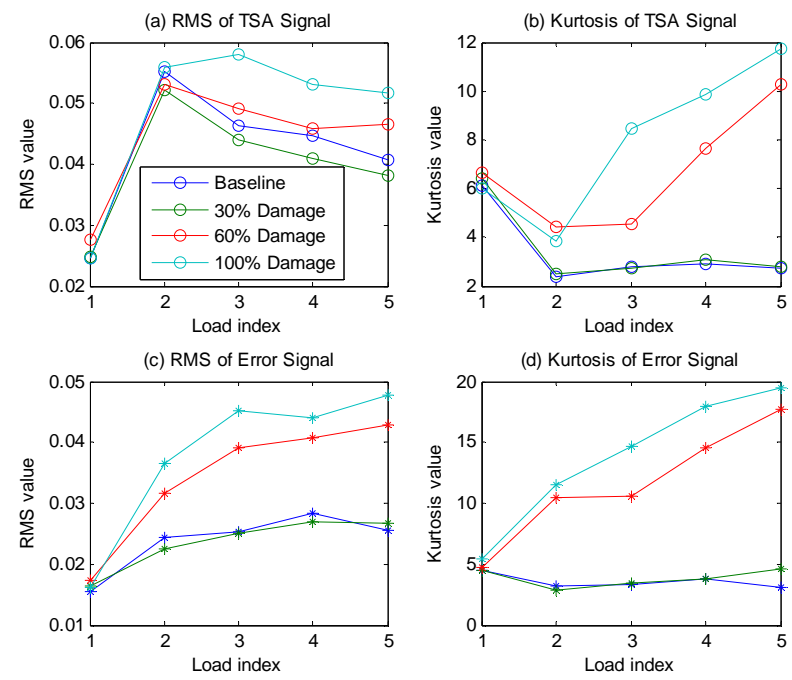

Figure 10. RMS and kurtosis value comparison of TSA and error signal $(\mathrm{L}=32, \mathrm{mu}=\mathbf{0 . 0 3 7 5}, \Delta=60)$.

Figure 10 illustrates the results obtained from another set of ALE parameters, the results for 30\% tooth damage is worse, while for higher severity faults the results is better.

\section{Conclusions}

In this study, ALE has been examined for the fault detection and diagnosis of a two stage helical gearbox based on noisy vibration signals. TSA is used to suppress the random noise firstly and then ALE is to reduce the continuous meshing components, which allows impulsive features shown by the time-frequency analysis representations to be highlighted effectively. In this way, the incipient tooth damage fault can be detected clearly.

\section{REFERENCES}

[1] R. M. Ramli, A. O. A. Noor and S. A. Samad, "A Review of Adaptive Line Enhancers for Noise Cancellation," Australian Journal of Basic and Applied Sciences, Vol. 6, No. 6, 2012, pp. 337-352.

[2] S. K. Lee and P. R. White, "The Enhancement of Impulsive Noise and Vibration Signals for Fault Detection in Rotating and Reciprocating Machinery," Journal of Sound and Vibration, Vol. 217, No. 3, 1998, pp. 485-505. doi:10.1006/jsvi.1998.1767

[3] J. R. Treichler, "Transient and Convergent Behaviour of the Adaptive Line Enhancer," IEEE Trans. Acousr. Speech Signal Processing. Vol. ASSP-27, pp. 53- 62.

[4] N. Sasaoka, K. Sumi, Y. Itoh and K. Fujii, “A New Noise Reduction System Based on ALE and Noise Reconstruction Filter," In proceeding of: International Symposium on Circuits and Systems, Kobe, 23-26 May 2005.

[5] J. R. Mohammed, M. S. Shafi, S. Imtiaz etc. "An Efficient Adaptive Noise Cancellation Scheme Using ALE and NLMS Filters," International Journal of Electrical and Computer Engineering, Vol. 2, No. 3, pp. 325-332.

[6] B. Widrow, J. R. Glover Jr, J. M. McCool, J. Kaunitz, C. S. Williams, R. H. Hearn, J. R. Zeidler, D. Eugene, Jr. and R. C. Goodlin, "Adaptive Noise Cancelling: Principles and Applications,” Proceedings of the IEEE, Vol. 63, No. 12, pp. 1692-1716.

[7] S. Haykin, “Adaptive Filter Theory,” Prentice Hall, 2002.

[8] S. R. Diniz, P., 2008. Adaptive Filtering: Algorithms and Practical Implementation, Springer.

[9] W. T. Wu, J. Lin, S. B. Han and X. H. Ding, "Time domain averaging based on fractional delay filter," $\mathrm{Me}$ chanical Systems and Signal Processing, Volume 23, No. 5, 2009, pp. 1447-1457. doi:10.1016/j.ymssp.2009.01.017

[10] J. R. Mohammed, "A New Simple Adaptive Noise Cancellation Scheme Based on ALE and NLMS Filter," Annual Conf. Communication, Networks, Services Research, May 2007, pp. 245-254. 